Boise State University

ScholarWorks

Kinesiology Faculty Publications and

Presentations

Department of Kinesiology

$3-2020$

\title{
A 4-Year Profile of Game Demands in Elite Women's Division I College Basketball
}

Lynda B. Ransdell

Northern Arizona University

Teena Murray

University of Louisville

Yong Gao

Boise State University

Paul Jones

University of Louisville

Dierdra Bycura

Northern Arizona University 


\title{
A 4-Year Profile of Game Demands in Elite Women's Division I College Basketball
}

\author{
Lynda B. Ransdell, ${ }^{1}$ Teena Murray, ${ }^{2}$ Yong Gao, ${ }^{3}$ Paul Jones, ${ }^{2}$ and Dierdra Bycura ${ }^{1}$ \\ ${ }^{1}$ Northern Arizona University, College of Health and Human Services, Flagstaff, Arizona; ${ }^{2}$ Department of Athletics, University of \\ Louisville, Louisville, Kentucky; and ${ }^{3}$ Department of Kinesiology, Boise State University, Boise, Idaho
}

\begin{abstract}
Ransdell, LB, Murray, T, Gao, Y, Jones, P, and Bycura, D. A 4-year profile of game demands in elite women's Division I college basketball. J Strength Cond Res 34(3): 632-638, 2020-Workload for a Division I women's collegiate basketball team (0.817 win percentage) was examined by: (a) season, (b) player position, and (c) game outcome (wins vs. losses). Female athletes ( $n=6$, mean $19.7 \pm 1.5$ years, at beginning of study) wore Catapult S5 units during 91.8\% of games over a 4-year period. Average PlayerLoad, PlayerLoad per minute (PL·min ${ }^{-1}$ ), high inertial movement analysis (high-IMA), and jumps were quantified using Catapult Openfield software (version 1.14.1+). Data were checked for normality and log- or square-root-transformed when they were non-normal. A series of linear mixed model analyses were conducted to detect differences in PlayerLoad, PL·min ${ }^{-1}$, high-IMA, and jumps by season, position, and game outcome. PL·min ${ }^{-1}$ and jumps data were not normal, so they were transformed, analyses were run; because there were no differences in findings, data are reported in original units to allow for comparisons with other studies. Cohen's $d$ and confidence intervals were provided as additional information about the strength of reported differences. The 3 most consistent findings were that across a 4-year period, jumps increased, PL·min ${ }^{-1}$ was higher in guards compared with posts, and high-IMA was higher in losses compared with wins. Other workload patterns were inconsistent, and inappropriate for making conclusive statements. Therefore, comparing jumps across multiple seasons, $\mathrm{PL} \cdot \mathrm{min}^{-1}$ by player position and high-IMA in losses are important; in addition, all data can be used to profile National Collegiate Athletic Association Division I women's basketball players and set game workload expectations.
\end{abstract}

Key Words: workload, catapult, athlete monitoring, wearable technology

\section{Introduction}

Division I National Collegiate Athletic Association (NCAA) college basketball is highly competitive, and female athletes playing at this level represent incredible talent. The Division I college basketball season is long and demanding. Teams play 30-35 regular season games (1-3 games per week) over a 5month period (November-March) before conference tournaments and the NCAA Tournament. Participating in postseason play can add another 10 games to reach the National Championship. Because of the length and intensity of the season, many coaches are now examining game demands (i.e., workload) in an effort to maximize performance.

Workload consists of whole body movements expressed as an accumulated load that combines rate of change in acceleration in 3 planes of movement: up/down (z), side/side (y), and forward/ backward (x); movements are ultimately scaled and expressed as AUs (20). Workload can be effectively assessed in basketball games and practices by using wearable tracking devices. Tracking of athletes who compete indoors combines gyroscopes (which determine the orientation of an athlete's body position), magnetometers (which

\footnotetext{
Address correspondence to Lynda Ransdell, Lynda.Ransdell@nau.edu.

Journal of Strength and Conditioning Research 34(3)/632-638

Copyright @ 2019 The Author(s). Published by Wolters Kluwer Health, Inc. on behalf of the National Strength and Conditioning Association. This is an open-access article distributed under the terms of the Creative Commons Attribution-Non Commercial-No Derivatives License 4.0 (CCBY-NC-ND), where it is permissible to download and share the work provided it is properly cited. The work cannot be changed in any way or used commercially without permission from the journal.
}

measure direction, similar to a digital compass) and accelerometers (which assess movement in 3 dimensions) into a noninvasive, small (96 $\times 52 \mathrm{~mm}$ ), wearable device (e.g., Catapult OptimEye S5, Melbourne, Australia) $(6,12,13)$. These devices are movement-based rather than position-based, and they collect data on accelerations, decelerations, jumps, and change of direction (38). Data provide a unique opportunity for a deep analysis of workload using sport- and positionspecific information.

Wearable devices are revolutionizing how teams plan training and manage athletes, and researchers have recently published reviews on the use of these wearable devices $(5,11,12)$ and the physiological demands and activity patterns of basketball players (34). Quantifying player workloads is fundamental to understanding the physical demands of a sport, and managing player risk (e.g., fatigue) and readiness (e.g., fitness) for sport. Specifically, workload monitoring during basketball games and practices is essential for (a) understanding sport-specific activities and metrics (e.g., PlayerLoad, jumps, and PlayerLoad per minute, an indicator of intensity of effort over time), (b) explaining acute changes in performance, (c) increasing understanding of training responses (and sport-specific training), and (d) facilitating effective planning and individualization of training (12). In addition, load monitoring during games can be used for technical and tactical evaluations (e.g., subbing and using specific players in specific situations), educating players and coaches about their performance, and developing databases of player profile metrics and elite performance models (18).

Profiling elite collegiate women's basketball players by season (longitudinally) gives coaches and researchers an idea of how players mature over time, and how workloads may increase with 
experience. Halperin et al. (16) suggest that longitudinal studies have greater external validity (e.g., findings are more like "real-life" and generalizable to other groups) and internal validity (e.g., more confidence that the intervention contributed to the outcome). Examining workload by game outcome and player position enables researchers to determine optimal workload, although opponent strength undoubtedly affects this value, and individual players may vary in their workload ability. Awareness of workload by game outcome is relevant, because coaches often tend to practice more after losses, and during losing seasons, which may increase fatigue and overuse injury risk. Ideally, knowledge of workload metrics by year, player position, and game outcome enables coaches and researchers to determine optimal workload.

Despite the well-established benefits of monitoring player workload, there is little information about the competition (game) workload demands of female basketball players. Scanlan et al. (29) compared workloads by player position and game quarter in state-level Australian women basketball players over 8 games to assess competition demands. Matthew and Delextrat (19) and Oba and Okuda (23) have also profiled the metabolic demands of women basketball players during competition (e.g., heart rate, blood lactate, and time motion analysis), but previous data involving female athletes were collected over short periods of time $(19,23,29)$, and only one study with female athletes has included data from a full season (24).

To our knowledge, no previous study has longitudinally quantified player workloads during games in NCAA Division I women's basketball players from the United States of America (USA) over a period of years, by player position, and by game outcome (e.g., wins vs. losses). Recent studies have called for more research on female basketball players (34), more longitudinal assessments of player workload $(7,31)$, assessments of how workload may change across multiple seasons or games $(12,29)$, and how workload varies by player position $(24)$ or game outcome $(13,30)$. If we understand game demands using longitudinal data, more effective short- and long-term planning can occur by season, player position, and game outcome.

Therefore, the purpose of this study was to quantify competition workloads: (a) of women's basketball players from an elite Division I program over 4 seasons (2014-2017), (b) by player position (guards vs. posts), and (c) by game outcome (wins vs. losses).

\section{Methods}

\section{Experimental Approach to the Problem}

A longitudinal, retrospective, and observational study design was used to examine 4 Catapult metrics by season of competition (2014-2017), player position (guards vs. posts), and game outcome (wins vs. losses). Sport science data were regularly collected by the sports performance coaches at a large urban university in the southeastern USA, thus data were available from 6 female basketball athletes during games over the past 4 seasons. Games in Division I women's basketball consisted of $2 \times 20$-minute halves in 2014-2015 and 2015-2016, and $4 \times 10$ minute quarters in 2016-2017 and 2017-2018.

Four (4) workload variables were selected for analysis, based on their common use in basketball, and their value in quantifying player workload for strength and conditioning staff members. These variables included: (a) average PlayerLoad (PL), in arbitrary units (AUs), an overall indicator of work performed during games that includes change of acceleration (forward, backward, sideways, and upward), divided by a scaling factor; (b) PlayerLoad per minute (PL·min ${ }^{-1}$ ), which indicates intensity of effort over time; (c) average high inertial movement analysis (high-IMA), which is a metric that combines acceleration, deceleration, change of direction, and free running, when the acceleration is greater than or equal to $3.50 \mathrm{~m} \cdot \mathrm{s}^{-1} \cdot \mathrm{s}^{-2}$, and (d) average jumps, which are calculated using an algorithm that estimates jump height based on hang time in the air (e.g., difference between the time the athlete leaves the ground until the athlete lands again).

Wearable devices have demonstrated acceptable reliability and validity in previous studies with soccer $(3,6)$, Australian football $(4)$, and during treadmill running (2). Nicolella et al. (22) found acceptable intra and interdevice accuracy and reliability for Catapult/PlayerLoad using standardized movements in multiple directions at 4 levels of peak acceleration. When the devices were compared with a reference accelerometer, effect sizes indicating differences were trivial to small.

\section{Subjects}

Six $(n=6)$ NCAA Collegiate Division 1 female basketball players competing in one of the top conferences in women's college basketball, the Atlantic Coast Conference, participated in the study. Athletes ranged in age from 18 to 22 years (mean age $19.7 \pm 1.5$ years at the start of the study). Although data were collected on all members of the roster, game data used for analysis were for the top 6 players on the team. From this group, 4 players had complete game data in 2014-2015 (2 were red-shirted), 6 players had complete data in 2015-2016, 5 in 2016-2017 (one was red-shirted), and 3 in 2017-2018 (3 graduated). These top 6 players were determined based on minutes played over the course of the season (starters, plus next highest minute earner), and data were compared across the 4 seasons for athletes, as is described in Table 1. Only guards and posts were compared in this study, because the university where data were collected categorizes players as guards or posts (centers plus forwards). Subjects signed informed consent forms to participate in the study when they started each new competitive season. Institutional review board approval was secured from the University of Louisville.

\section{Procedures}

The Catapult OptimEye S5, which was used to collect data, was placed in a supportive harness and positioned on the back, between the scapulae in a standardized location, before every game; equipment was removed immediately after the game (4). Players were assigned the same wearable device every game (22), by trained technicians, and the pre and postgame routines for unit distribution, activation, and collection were standardized. In addition, only data that corresponded to the start and end time of the game, excluding quarter or halftime breaks or time-outs, were included in the analysis (17). Catapult data were downloaded and analyzed using Catapult software (Openfield; Catapult Innovations, Melbourne, Australia), before being exported to a Microsoft Excel Spreadsheet (Redmond, WA).

\section{Statistical Analyses}

Mean and SDs were calculated for all descriptive and workload variables. Data are presented by season, player position (guards vs. posts), and game outcome (wins vs. losses). Normality of the 4 workload variables (PlayerLoad, PL· min $^{-1}$, high-IMAs, and jumps) was checked through Kolmogorov-Smirnov tests (33), and data were normalized through logarithmic or square root transformation if the distributions were not normal. Because of the data structure (i.e., multiple observations were nested within each subject then across multiple (4) seasons and/or game outcomes), a series of linear 
Table 1

Win-loss records across past four seasons, percentage of games monitored, and number of players monitored.

\begin{tabular}{lcccccc}
\hline Season & Losses (n) & Percentage (\%) & Wins (n) & Percentage (\%) & Total games (\% games monitored) & Players monitored (n) \\
\hline $2014-2015$ & 7 & 20.6 & 27 & 79.4 & $34(79 \%)$ & 4 \\
$2015-2016$ & 8 & 23.5 & 26 & 76.5 & $34(88 \%)$ & 6 \\
$2016-2017$ & 8 & 21.6 & 29 & 78.4 & $37(100 \%)$ & 5 \\
$2017-2018$ & 3 & 7.7 & 36 & 92.3 & $39(100 \%)$ & 3 \\
4-year average & 6.5 & 18.3 & 28.3 & 81.7 & $36(91.8 \%)$ & \\
\hline
\end{tabular}

mixed model analyses were conducted (24). Linear mixed models were used to determine whether there were differences in the 4 workload variables (i.e., PlayerLoad, PL $\min ^{-1}$, high-IMAs, and jumps) by season, player position, and game outcome.

Normality tests indicated that variables PlayerLoad and highIMAs were normally distributed overall, by season, by game outcome, and by position, $p \geq 0.05$, but PL $\cdot \mathrm{min}^{-1}$ and jumps were not. Therefore, these 2 variables were normalized (PL $\mathrm{min}^{-1}$ through logarithmic transformation and jumps through square root transformation). However, the linear mixed model analyses on normalized and non-normalized PL·min ${ }^{-1}$ and jumps data revealed similar results; therefore, results using original PL $\cdot \min ^{-1}$ and jumps data are reported in this paper to facilitate future data comparisons.

Where appropriate, Cohen's $d$ (9), with $0.2-0.49$ as small, 0.5-0.79 medium, and 0.8 and above large, along with $95 \%$ confidence intervals, were reported. All statistical analyses were conducted using SPSS 25 and significance level was set at 0.05 .

\section{Results}

\section{Team Success Statistics and Player Monitoring Information}

The win-loss records, number of games monitored, and number of players monitored for the 4 seasons (from 2014 to 2017) are presented in Table 1 . Win percentages were consistently high across the 4-year period, with the highest win percentage occurring in 2017-2018, when the team advanced to the final 4 of the NCAA Women's Division I Championship.

\section{Results by Season}

Table 2 presents aggregate average PlayerLoad, PL $\cdot \mathrm{min}^{-1}$, highIMA, and jumps by season. Linear mixed model analyses revealed a significant main effect on 3 of the 4 variables measured. Average 4-year PlayerLoad was significantly different across seasons $(p=$ $0.002)$. Further analyses revealed that PlayerLoad was significantly higher in 2015-2016 $(p=0.024, d=0.37)$ and 2016-2017 ( $p=0.047, d=0.30$ ) than in 2017-2018. In addition, PlayerLoad was lower in 2014-2015 than in 2015-2016 ( $p$ $=0.001, d=0.58)$ and 2016-2017 ( $p=0.002, d=0.48$. Average 4 -year $\mathrm{PL} \cdot \mathrm{min}^{-1}$ was also significantly different across seasons, $p<0.001$, where PL· $\min ^{-1}$ in 2016-2017 was significantly lower than in the other 3 seasons, and effect sizes for all of these comparisons were large $(d=0.81-1.03)$. A significant main effect of season on jumps was observed $(p=0.002)$ where average jumps in the first season (2014-2015) were statistically lower than those in 2016-2017 ( $p=0.022, d=0.30)$ and 2017-2018 $(p=0.001, d=0.55)$, and average jumps in the second season (2015-2016) were also significantly lower than those in 2017-2018 ( $p=0.01, d=0.44)$. There were no statistical differences in high-IMAs across the 4 seasons, $p=0.114$.

\section{Results by Player Position}

Table 3 presents game averages by player position, and by season. In general, it seems as if guards had significantly higher PlayerLoad than posts $(p=0.004)$; however, a closer look indicates a significant season-by-position interaction $(p<0.001)$. There

Table 2

Game averages by season. ${ }^{*} \dagger$

\begin{tabular}{|c|c|c|c|c|}
\hline Season & Player load & Player load/min & High IMA & Jumps \\
\hline 2014-2015 & $587.9 \pm 165.3$ & $7.3 \pm 1.0$ & $47.1 \pm 16.5$ & $81.9 \pm 24.0$ \\
\hline 2015-2016 & $682.7 \pm 162.9$ & $7.4 \pm 1.3$ & $54.2 \pm 20.3$ & $85.8 \pm 26.9$ \\
\hline 2016-2017 & $678.1 \pm 198.0$ & $6.4 \pm 1.1$ & $52.1 \pm 19.4$ & $92.4 \pm 39.2$ \\
\hline 2017-2018 & $626.1 \pm 131.1$ & $7.4 \pm 0.9$ & $52.3 \pm 14.2$ & $99.7 \pm 38.2$ \\
\hline 4-year average & $655.6 \pm 173.2 \pm \S$ & $7.1 \pm 1.2 \pm \S$ & $52.1 \pm 18.5$ & $89.8 \pm 33.4 \pm \S$ \\
\hline Season comparisons & $\begin{array}{c}\text { Cohen's } \\
d(95 \% \text { Cl for } d) \\
\end{array}$ & $\begin{array}{c}\text { Cohen's } \\
d(95 \% \text { Cl for } d) \\
\end{array}$ & $\begin{array}{c}\text { Cohen's } \\
d(95 \% \text { Cl for } d) \\
\end{array}$ & $\begin{array}{c}\text { Cohen's } \\
d(95 \% \text { Cl for } d) \\
\end{array}$ \\
\hline 2014-2015 vs. 2015-2016 & $0.58(0.32$ to 0.83$) \S$ & $0.03(-0.22$ to 0.28$)$ & 0.37 (0.12 to 0.62$)$ & $0.15(-0.1$ to 0.4$)$ \\
\hline 2014-2015 vs. 2016-2017 & 0.48 (0.22 to 0.74$) \S$ & 0.91 (0.64 to 1.17$) \S$ & 0.27 (0.02 to 0.53$)$ & $0.30(0.04$ to 0.56$) \|$ \\
\hline 2014-2015 vs. 2017-2018 & $0.26(-0.03$ to 0.54$)$ & $0.13(-0.16$ to 0.41$)$ & 0.34 (0.06 to 0.63$)$ & 0.55 (0.26 to 0.83$) \S$ \\
\hline 2015-16 vs. 2016-2017 & $0.03(-0.18$ to 0.23$)$ & 0.81 (0.6 to 1.02$) \S$ & $0.10(-0.1$ to 0.31$)$ & $0.20(-0.01$ to 0.4$)$ \\
\hline 2015-2016 vs. 2017-2018 & 0.37 (0.13 to 0.61$) \|$ & $0.07(-0.17$ to 0.31$)$ & $0.10(-0.14$ to 0.34$)$ & 0.44 (0.2 to 0.68$) \Phi$ \\
\hline 2016-2017 vs. 2017-2018 & $0.30(0.05$ to 0.54$) \|$ & 1.03 (0.77 to 1.28$) \S$ & $0.01(-0.23$ to 0.25$)$ & $0.19(-0.06$ to 0.43$)$ \\
\hline
\end{tabular}

*IMA = inertial movement analysis; $\mathrm{Cl}=$ confidence interval.

†Numbers in upper panel are presented as mean $\pm S D$; numbers in lower panel represents effect size Cohen's $d$ (95\% Cls for $d$ ); Cohen's $d$ effect sizes $=0.2-0.49$ is small, $0.5-0.79$ is medium, and 0.8 or higher is large.

$\ddagger$ Significant main effect of season.

§Significant differences between comparisons, where $p<0.005$

\|Significant differences between comparisons, where $p<0.05$.

ฯSignificant differences between comparisons, where $p<0.01$. 


\begin{tabular}{|c|c|c|c|c|c|c|}
\hline \multirow[b]{2}{*}{ Year } & \multicolumn{2}{|c|}{ Player load } & \multirow{2}{*}{$\begin{array}{l}\text { Cohen's } \\
d(95 \% \text { Cl for } d)\end{array}$} & \multicolumn{2}{|c|}{ Player load $\cdot \min ^{-1}$} & \multirow{2}{*}{$\begin{array}{c}\text { Cohen's } \\
d(95 \% \text { Cl for } d)\end{array}$} \\
\hline & Guard & Post & & Guard & Post & \\
\hline $2014-2015$ & $531.2 \pm 140.0$ & $614.8 \pm 170.5$ & $0.52(0.06$ to 0.96$) \neq$ & $7.5 \pm 0.9$ & $7.2 \pm 1.0$ & $0.34(-0.11$ to 0.78$)$ \\
\hline 2015-2016 & $669.9 \pm 178.3$ & $695.6 \pm 145.5$ & $0.16(-0.12$ to 0.44$)$ & $7.6 \pm 1.4$ & $7.1 \pm 1.2$ & $0.38(0.1$ to 0.66$) \S$ \\
\hline $2016-2017$ & $751.7 \pm 175.1 \#$ & $633.2 \pm 198.4$ & $0.62(0.31$ to 0.93$) \S$ & $6.9 \pm 1.0$ & $6.1 \pm 1.0$ & $0.80(0.48$ to 1.11$) \S$ \\
\hline 2017-2018 & $676.4 \pm 121.8 \#$ & $529.9 \pm 88.1$ & $1.31(0.86$ to 1.74$) \S$ & $7.9 \pm 0.8$ & $6.6 \pm 0.5$ & $1.74(1.26$ to 2.2$) \S$ \\
\hline \multirow[t]{2}{*}{ 4-year average } & $676.8 \pm 170.9$ & $637.2 \pm 173.3$ & $0.23(0.06$ to 0.4$) \S \|$ & $7.5 \pm 1.2 \#$ & $6.7 \pm 1.1$ & 0.68 (0.51 to 0.85$) \S \mid$ \\
\hline & \multicolumn{2}{|c|}{ High IMA } & Cohen's & \multicolumn{2}{|c|}{ Jumps } & Cohen's \\
\hline Year & Guard & Post & $d(95 \%$ CI for $d)$ & Guard & Post & $d(95 \%$ CI for $d)$ \\
\hline $2014-2015$ & $38.0 \pm 16.2$ & $51.4 \pm 14.9$ & $0.87(0.40$ to 1.32$) \S$ & $86.4 \pm 22.4$ & $79.8 \pm 24.7$ & $0.27(-0.17$ to 0.72$)$ \\
\hline 2015-2016 & $49.2 \pm 22.8$ & $59.2 \pm 15.9$ & $0.51(0.22$ to 0.79$) \S$ & $85.0 \pm 25.2$ & $86.6 \pm 28.6$ & $0.06(-0.22$ to 0.34$)$ \\
\hline 2016-2017 & $58.5 \pm 20.0 \#$ & $48.2 \pm 18.1$ & $0.55(0.24$ to 0.86$) \S$ & $110.8 \pm 37.7 \#$ & $81.2 \pm 35.9$ & $0.81(0.49$ to 1.12$) \S$ \\
\hline 2017-2018 & $50.6 \pm 14.3$ & $55.7 \pm 13.6$ & $0.36(-0.05$ to 0.76$)$ & $94.5 \pm 43.4$ & $109.8 \pm 22.6$ & $0.41(0$ to 0.81$) \Phi$ \\
\hline 4-year average & $50.7 \pm 20.2$ & $53.3 \pm 16.9$ & $0.14(-0.03$ to 0.31$) \S \|$ & $94.1 \pm 35.3$ & $86.1 \pm 31.4$ & $0.24(0.08$ to 0.41$) \S \|$ \\
\hline
\end{tabular}

*IMA = inertial movement analysis; $\mathrm{Cl}=$ confidence interval.

†Numbers are presented as mean $\pm S D$, and effect size Cohen's $d(95 \%$ Cls for $d$; Cohen's $d$ effect sizes $=0.2-0.49$ is small, $0.5-0.79$ is medium, and 0.8 or higher is large.

¥Significant differences between comparisons, where $p<0.01$.

$\S$ Significant differences between comparisons, where $p<0.005$.

\|Significant season by player's position interaction.

-Significant differences between comparisons, where $p<0.05$

\#Significant differences by season, where $p<0.01$

were many inconsistencies in the data by player position and by season. Specifically, there were no PlayerLoad differences between guards and posts in 2015-2016 season $(p=0.247, d=$ $0.16)$, guards had significantly lower PlayerLoad than posts in 2014-2015 season $(p=0.008, d=0.52)$, and such differences reversed in 2016-2017 $(p<0.001, d=0.62)$ and 2017-2018 ( $p$ $<0.001, d=1.31$ ) when guards had significantly higher PlayerLoad than posts.

Table 3 also shows that over the 4 seasons, guards had statistically higher PL $\cdot \min ^{-1}$ than posts $(p<0.001, d=0.68)$, and this was consistent across 2015-2016, 2016-2017, and 2017-2018 seasons $(p<0.001)$; however, in 2014-2015, no difference in $\mathrm{PL} \cdot \mathrm{min}^{-1}$ between guards and posts was observed $(p=0.071$, $d=0.34)$.

When high-IMA was examined over 4 seasons, posts had higher high-IMAs than guards $(p=0.003, d=0.14)$. Further analysis revealed a significant season-by-position interaction $(p<$ 0.001 ) which showed inconsistent results over time. Guards had significantly lower high-IMAs than posts in 2014-2015 $(p<$ $0.001, d=0.87)$ and 2015-2016 $(p<0.001, d=0.51)$, but higher high-IMAs than posts in 2016-2017 $(p<0.001, d=0.55)$ and similar high IMAs to posts in 2017-2018 $(p=0.12)$.

When 4 seasons of jumps were examined, guards registered more jumps than posts $(p<0.001, d=0.24)$. On further analysis, results were inconsistent over time. Specifically, no statistical differences were observed in jumps between guards and posts in 2014-2015 ( $p=0.35, d=0.27)$ and 2015-2016 ( $p=0.705, d=$ $0.06)$; guards had significantly higher jumps than posts in 2016-2017 ( $p<0.001, d=0.81)$, and significantly lower jumps than posts in 2017-2018 ( $p=0.015, d=0.41)$.

\section{Results by Game Outcome (Wins vs. Losses)}

Table 4 presents PlayerLoad, PL·min ${ }^{-1}$, high-IMA and jumps by game outcome. Linear mixed models revealed no statistical differences in PlayerLoad $(p=0.095), \mathrm{PL} \cdot \mathrm{min}^{-1}(p=0.654)$, and jumps $(p=0.766)$ by game outcome. However, a main effect of game outcome on high-IMAs was observed $(p=0.015)$ : players consistently experienced significantly higher high-IMAs in games that were lost compared with those that were won, which was much more evident during the most successful season (2017-2018), Cohen's $d=1.03$.

\section{Discussion}

The most important findings of the study were that: (a) averages, effect sizes, and variability for PlayerLoad, PL $\cdot \mathrm{min}^{-1}$, high-IMA, and jumps were established for this sample, such that a range of values for a player profile now exists; (b) consistent findings include an increase in jumps in the last 2 seasons compared with the first 2 seasons, higher PL $\cdot \mathrm{min}^{-1}$ in guards compared with forwards in the last 3 seasons measured, and higher high-IMA in losses compared with wins; (c) with the exception of the consistent workload patterns mentioned above, other values were inconsistent across season, by player position, and by game outcome.

Previous research has not profiled workload variables in a sample of NCAA Division I women's basketball players during competition, nor has previous research in men or women examined workload longitudinally over multiple seasons. Because values for PlayerLoad, PL·min ${ }^{-1}$, high-IMA, and jumps fell within a consistent range, these values can be used to (a) establish a range of game metrics for the 4 measured variables by individual player, team, or player position, (b) set game expectations, and (c) establish practices and strength and conditioning sessions that approximate these values, as part of a periodized program, so that practice intensity matches that of games. The consistency in these workload values may also point to the importance of building a systematic, individualized, data-based, and long-term plan for athletes.

In one of the few studies to report data that can be compared to ours, Fox et al. (14) used wearable devices on 15 semiprofessional male basketball players and reported lower PlayerLoad during competition $(448 \pm 118)$ than during games-based training (624 $\pm 113, d=1.54)$ or physical conditioning $(632 \pm 139, d=1.36)$. 
Table 4

Game averages by outcome (wins and losses). ${ }^{*} \dagger$

\begin{tabular}{|c|c|c|c|c|c|c|}
\hline \multirow[b]{2}{*}{ Year } & \multicolumn{2}{|c|}{ Player load } & \multirow{2}{*}{$\begin{array}{c}\text { Cohen's } \\
d(95 \% \text { Cl for } d)\end{array}$} & \multicolumn{2}{|c|}{ Player load $\min ^{-1}$} & \multirow{2}{*}{$\begin{array}{c}\text { Cohen's } \\
d(95 \% \text { Cl for } d) \\
\end{array}$} \\
\hline & $\mathbf{L}$ & W & & $\mathbf{L}$ & W & \\
\hline 2014-2015 & $610.8 \pm 183.2$ & $580.9 \pm 160.2$ & 0.18 ( -0.31 to 0.67$)$ & $7.2 \pm 0.9$ & $7.4 \pm 1.0$ & $0.24(-0.25$ to 0.73$)$ \\
\hline 2015-2016 & $693.4 \pm 180.1$ & $679.2 \pm 157.4$ & $0.09(-0.24$ to 0.41$)$ & $7.3 \pm 1.4$ & $7.4 \pm 1.3$ & $0.1(-0.23$ to 0.42$)$ \\
\hline 2016-2017 & $699.5 \pm 217.8$ & $672.1 \pm 192.5$ & $0.14(-0.22$ to 0.5$)$ & $6.3 \pm 1.2$ & $6.4 \pm 1.1$ & $0.11(-0.25$ to 0.47$)$ \\
\hline 2017-2018 & $718.9 \pm 88.7$ & $617.4 \pm 131.4$ & 0.79 (0.09 to 1.47$)$ & $7.5 \pm 0.8$ & $7.4 \pm 1.0$ & $0.09(-0.6$ to 0.77$)$ \\
\hline \multirow[t]{2}{*}{ 4-year average } & $682.4 \pm 190.0$ & $648.7 \pm 168.1$ & $0.20(-0.01$ to 0.4$)$ & $6.9 \pm 1.3$ & $7.1 \pm 1.2$ & $0.13(-0.07$ to 0.33$)$ \\
\hline & \multicolumn{2}{|c|}{ High IMA } & Cohen's & \multicolumn{2}{|c|}{ Jumps } & Cohen's \\
\hline Year & $\mathbf{L}$ & W & $d(95 \% \mathrm{Cl}$ for $d)$ & $\mathbf{L}$ & $\mathbf{W}$ & $d(95 \% \mathrm{Cl}$ for $d)$ \\
\hline 2014-2015 & $51.0 \pm 16.7$ & $45.9 \pm 16.4$ & $0.31(-0.18$ to 0.8$)$ & $82.5 \pm 22.3$ & $81.7 \pm 24.7$ & $0.03(-0.46$ to 0.52$)$ \\
\hline 2015-2016 & $58.0 \pm 22.4$ & $52.9 \pm 19.4$ & $0.25(-0.08$ to 0.58$)$ & $85.7 \pm 26.0$ & $85.9 \pm 27.3$ & $0.01(-0.32$ to 0.33$)$ \\
\hline 2016-2017 & $53.5 \pm 20.8$ & $51.7 \pm 19.1$ & $0.09(-0.27$ to 0.45$)$ & $87.8 \pm 40.1$ & $93.7 \pm 39$ & $0.15(-0.21$ to 0.51$)$ \\
\hline 2017-2018 & $65.2 \pm 10.7$ & $51.1 \pm 13.9$ & 1.03 (0.33 to 1.72 ) & $109.8 \pm 38.7$ & $98.8 \pm 38.2$ & 0.29 (-0.4 to 0.97$)$ \\
\hline 4-year average & $55.8 \pm 20.4$ & $51.1 \pm 17.9$ & 0.26 (0.05 to 0.46$) \neq \S$ & $87.7 \pm 32.1$ & $90.4 \pm 33.8$ & $0.08(-0.12$ to 0.28$)$ \\
\hline
\end{tabular}

${ }^{*} \mathrm{MA}=$ inertial movement analysis; $\mathrm{Cl}=$ confidence interval.

†Numbers are presented as mean $\pm S D$, and effect size Cohen's $d(95 \%$ Cls for $d$ ); Cohen's $d$ effect sizes $=0.2-0.49$ is small, $0.5-0.79$ is medium, and 0.8 or higher is large ‡Significant main effect of outcome.

$\S$ Significant differences between comparisons, where $p<0.05$.

Heishman et al. (17) and Svilar et al. (35) examined elite male basketball players (Division I; $n=10 ; 20.9$ years, and Euroleague; $n=13 ; 25.7$ years, respectively), and reported that mean PlayerLoad during practices was $338 \pm 38$, and $315 \pm 90$, respectively. Female PlayerLoad values from the present study were similar to male PlayerLoad values during games-based training and physical conditioning (14), and notably higher than that of men during practices $(17,35)$ and in one study, games $(14)$. What is unclear from the previous studies with male athletes $(14,17,35)$, is the type of activities conducted during practice or the level of competition faced in game play. It is possible that these studies with men were done during lower-level game play or practice, that more substitutes or rest breaks were available (and playing time differences may explain workload differences), or that game and practice tactics and strategies were different.

Few data exist that profile jumping during competition and practice for female athletes. Of the women's data that were reported using video analysis (34), a high of $43 \pm 5$ jumps was reported in the State Basketball League in Australia (29) and a low of $16 \pm 6$ jumps was reported in USA Division II NCAA Collegiate basketball championships (21). Differences between data from the present study and other women's data may be related to variations in level of competition (practice vs. game play), length of game play, playing time differences (which may change across a college career), or style of play (27). It is also possible that different techniques used to collect data (i.e., video vs. accelerometer) may explain variation in results (28). More research is needed to profile differences in jumps during practices and games, using consistent technology, across various levels of competition, and by age and sex.

In the present study, although many workload values did not demonstrate a consistent pattern, 3 important patterns were noted across the sample. First, the number of jumps increased significantly from the first 2 years of monitoring (2014-2015 and 2015-2016) to the last 2 years (2016-2017 and 2017-2018). An increase in the number of game-based jumps over time is indicative of high-level longitudinal athletic performance in basketball $(8,32)$. Theoretically, if an athlete is jumping more in games as her career progresses, performance should improve. This significant increase in the number of game-based jumps over time is consistent with the fact that the win-loss record for the team over the 4-year period was consistently near $80 \%$, and the last year win percentage was the highest. So, training to jump and monitoring number of jumps during games may have a positive effect on performance, and teams can monitor jumps over time to see whether increases in jumps are related to improvements in performance.

Second, average PL $\cdot \mathrm{min}^{-1}$ was higher in guards compared with posts in all but the first year of data collection. Guards often cover large areas of the court, offensively and defensively, (e.g., dribbling the ball up the court, breaking a press, or implementing a press), making this finding consistent with previous literature using time motion analysis that concluded that guards tend to have more highintensity activity than posts $(10,12,15)$. In addition, previous research with male basketball athletes has shown that compared with posts, guards typically have faster acceleration, agility, and speed ( 5 - and 10 -m performances) $(1,25,27,31)$, and they may be able to accelerate easier with less applied force because of their lower body mass $(12,26)$. Theoretically, these positional baseline differences in acceleration, agility, and speed should be related to guards recording higher PL· $\mathrm{min}^{-1}$ values compared with posts (36).

Third, high-IMA was statistically higher during losses compared with wins. The idea that high-IMA was higher during losses could indicate increased workload to regain a lead after a deficit, or a lack of "flow" that can occur in stressful situations (37). Interestingly, high-IMA varied significantly by year and player position. Some years it was higher for posts and other years it was higher for guards. This may indicate that high-IMA varies according to the style of play of the team.

There were other interesting fluctuations in workload by position. For example, PlayerLoad was significantly higher for posts than guards in 2014-2015, and it was significantly higher for guards compared with posts in 2016-2017 and 2017-2018. High inertial movement analysis was significantly higher for posts compared with guards in 2014-2015 and 2015-2016, but higher for guards compared with posts in 2016-2017. These fluctuations in workload variables could be due to player maturation over time or strength of schedule (with strong opposing athletes matching up with different positions). 
In addition to statistically significant differences, nonsignificant differences are also worth noting. For example, PlayerLoad and jumps were similar regardless of game outcome. Consistent profiles of these values across the team, regardless of player position, year, or game outcome, may point to the ability of coaches of elite teams to motivate athletes to put forth consistent game effort, or it may point to an ability to recruit athletes with similar athletic profiles. The fact that many of the workload values were not statistically different across season indicates that all athletes are working similarly, regardless of player position. Intuitively, it makes sense that when teams are all working hard together, they have the most potential for success.

Although this is the first study to report longitudinal (4-year), descriptive data on a Division I elite women's basketball team, it is not without limitations. The sample size is relatively small, and only one team is included. Results are specific to this team, and based on differences in player ability, fitness, and game scores, which may vary by opponent ability and tactical game strategies. Therefore, study results may not be generalizable to other levels of women's teams (high school, club, other collegiate levels: Division II and III, or professional teams such as the Women's National Basketball Association or WNBA), or to men who play basketball.

Finally, because data collection and analysis vary by software and analysis sought, findings may be difficult to compare. Nicollella et al. (22) recommends that researchers should work to standardize device placement strategies, metrics used, and the methods used to calculate results.

In the future, researchers should continue to examine longitudinal training, practice and game habits of elite athletes, and they should seek to link workload metrics with injury rates and game statistics (e.g., free throw or field goal percentage). It would also be interesting to examine whether game demands, performance, and injury rates differ when the game is played in halves or quarters. Finally, researchers and strength and conditioning coaches should use data collected to develop the optimal periodization scheme, and level of work expected in practices and games-by individual athlete and as a team.

\section{Practical Applications}

Performance or strength and conditioning coaches working with basketball teams can use these findings in a variety of ways. Working backwards from a solid understanding of game workloads, performance coaches can work with coaching staff to effectively organize daily training (practices) to optimize player readiness for game day. In addition to daily loads, ideally performance coaches will organize weekly and monthly loading plans by including volume and intensity, to ensure progressions and practice demands are appropriate and effective for optimizing performance for each player.

Another way to use game monitoring is to correlate gameday workload metrics with physiological measures related to recovery (e.g., creatine kinase, subjective rating of muscle soreness, rating of perceived exertion, or perceptive recovery quality.

A more ambitious possibility related to daily load monitoring is the ability to correlate key performance metrics with key basketball box score metrics. For example, coaches could ask whether there is a relationship between multiple day (2-, 3-, and 4-day) PlayerLoads leading in to games, and subsequent game-day shooting percentage. It is important to monitor practice loads and to ensure that weekly totals are planned according to an effective progression early in the season, ensuring the acute loads align well with chronic loads.

Similarly, data can be used to identify which variables best predict optimal player characteristics, or optimal player characteristics for critical moments within games. Finally, as previously discussed, coaches can use this information to identify critical performance benchmarks, develop practice plans that align with key practice outcomes, and model testing and training protocols after one of the top women's basketball programs in the USA.

In conclusion, the 3 most consistent findings were that jumps increased in the last 2 years compared with the first 2 years, PL $\cdot \min ^{-1}$ was higher in guards compared with posts in 3 of the seasons assessed, and high-IMA was higher in losses compared with wins. Other workload patterns examined were inconsistent, unpredictable by season, and they were not adequate for making conclusive statements. Therefore, paying attention to jumps, PL. $\mathrm{min}^{-1}$, and high-IMA relative to season, player position and game outcomes, respectively, is important. For example, monitoring jumps during game play and over an athlete's career, may provide benchmarks and motivate athletes to jump more during games, which ultimately could lead to enhanced performance. It is also possible that monitoring PL $\cdot \mathrm{min}^{-1}$ by player position may motivate forwards to produce higher levels of PL $\cdot \mathrm{min}^{-1}$, or facilitate friendly competition to increase movement intensity during game play. Finally, recognizing that high-IMA is higher during losses compared to wins, it makes sense to encourage athletes to focus on flow or natural movement during a game as compared to tense, less coordinated movements that sometimes occur when an athlete is playing "not to lose" rather than "playing to win."

The longitudinal team averages can best be used to profile athletes and set game workload expectations for NCAA Division I women's basketball players. Clearly, objective game data are essential for advancing the understanding of game demands, allowing for the implementation of enhanced player development and preparation strategies. Equally important, an understanding of game workloads and specific intensity metrics are essential for managing noncontact injury risk. This information should assist performance coaches and basketball coaches alike in developing improved player monitoring systems during the competitive season.

\section{Acknowledgments}

The authors are appreciative of the philosophy of the university's women's basketball team to support conducting research to enhance the health and performance of student-athletes. No sources of funding were sought for this publication. Results of the present study do not constitute an endorsement of this product by the authors or the National Strength and Conditioning Association. 


\section{References}

1. Abdelkrim NB, Chaouachi A, Chamari K, Castagna C. Positional role and competition-level differences in elite level men's basketball players. J Strength Cond Res 24: 1346-1355, 2010.

2. Barrett S, Midgley A, Lovell R. PlayerLoad ${ }^{\mathrm{TM}}$ : Reliability, convergent validity and influence of unit position during treadmill running. Int $J$ Sports Physiol Perf 9: 945-952, 2014.

3. Barrett S, Midgley AW, Towlson C, et al. Within-match PlayerLoad patterns during a simulated soccer match: Potential implications for unit positioning and fatigue management. Int J Sports Physiol Perf 11: 135-140, 2016.

4. Boyd LJ, Ball K, Aughey RJ. The reliability of MinimaxX accelerometers for measuring physical activity in Australian football. Int J Sports Physiol Perf 6: 311-321, 2011.

5. Cardinale M, Varley MC. Wearable training monitoring technology: Applications, challenges and opportunities. Int J Sports Physiol Perform 12: S255-S262, 2017.

6. Casamichana D, Castellano J, Calleja-Gonzalez J, San Roman J, Castagna C. Relationship between indicators of training load in soccer players. J Strength Cond Res 27: 369-374, 2013.

7. Castagna C, Abt G, Manzi V, et al. Effect of recovery mode on repeated sprint ability in young basketball players. J Strength Cond Res 22: 923-929, 2008.

8. Claudino JG, Cronin J, Mezencio B, et al. The countermovement jump to monitor neuromuscular status: A meta-analysis. J Sci Med Sport 20: 397-402, 2017.

9. Cohen J. Statistical Power Analysis for the Behavioral Sciences (2nd ed.). Hillsdale, NJ: Lawrence Erlbaum Associates, 1988. pp. 24-26.

10. Delextrat A, Badiella A, Saavedra V, et al. Match activity demands of elite Spanish female basketball players by playing position. Int J Perform Anal Sport 15: 687-703, 2015.

11. Dellaserra CL, Gao Y, Ransdell LB. Use of integrated technology in team sports: A review of opportunities, challenges and future directions for athletes. J Strength Cond Res 28: 556-573, 2014.

12. Edwards T, Spiteri T, Piggott B, et al. Monitoring and managing fatigue in basketball. Sports (Basel) 6: 19, 2018.

13. Fox JL, Scanlan AT, Stanton R. A review of player monitoring approaches in basketball: Current trends and future directions. J Strength Cond Res 31: 2021-2029, 2017.

14. Fox JL, Stanton R, Scanlan AT. A comparison of training and competition demands in semiprofessional male basketball players. Res Q Exerc Sport 89: 103-111, 2018.

15. Grosgeorge B. Observation and Training in Team Sports. Paris, France: INSEP-Publications, 1990.

16. Halperin I, Vigotsky AD, Foster C, Pyne DB. Strengthening the practice of exercise and sport-science research. Intl J Sports Physio Perform 13: 127-134, 2018.

17. Heishman AD, Curtis MA, Saliba E, et al. Non-invasive assessment of internal and external player load: Implications for optimizing athletic performance. J Strength Cond Res 32: 1280-1287, 2018.

18. Hughes MD, Franks IM. Notational Analysis of Sport: Better Systems for Improving Coaching and Performance (2nd ed.). London, United Kingdom: Routledge, 2004.

19. Matthew D, Delextrat A. Heart rate, blood lactate concentration, and time-motion analysis of female basketball players during competition. J Sport Sci 27: 813-821, 2009.
20. Montgomery PG, Pyne DB, Minahan CL. The physical and physiological demands of basketball training and competition. Int J Sports Physio Perform 5: 75-86, 2010.

21. Narazaki K, Berg K, Stergiou N, Chen B. Physiological demands of competitive basketball. Scand J Med Sci Sports 19: 425-432, 2009.

22. Nicolella DP, Torres-Ronda L, Saylor KJ, Schelling X. Validity and reliability of an accelerometer-based player tracking device. PLoS One 13: e0191823, 2018.

23. Oba W and Okuda T. A cross-sectional comparative study of movement distances and speed of the players and ball in a basketball game. Int J Sport Health Sci 6: 203-212, 2008.

24. Paulauskas H, Kreivyte R, Scanlan AT, et al. Monitoring workload in elite female basketball players during the in-season phase: Weekly fluctuations and effect of playing time. Int J Sports Physiol Perform 14: 941-948, 2019.

25. Puente C, Abian-Vicen J, Arces F, Lopez R, Del Coso J. Physical and physiological demands of experienced male basketball players during a competitive game. J Strength Cond Res 31: 956-962, 2016.

26. Ransdell LB, Wells CL. Sex differences in athletic performance. Women Sport Phys Activity J 8: 55-79, 1999.

27. Scanlan A, Dascombe BJ, Kidcaff AP, Peucker JL, Dalbo VJ. Genderspecific activity demands experienced during semiprofessional basketball game play. Int J Sports Physiol Perf 10: 618-625, 2015.

28. Scanlan A, Dascombe B, Reaburn P. A comparison of the activity demands of elite and sub-elite Australian men's basketball competition. J Sports Sci 29: 1153-1160, 2011.

29. Scanlan AT, Dascombe BJ, Reaburn P, Dalbo VJ. The physiological and activity demands experienced by Australian female basketball players during competition. J Sci Med Sport 15: 341-347, 2012.

30. Scanlan AT, Tucker PS, Dascombe BJ, et al. Fluctuations in activity demands across game quarters in professional and semiprofessional male basketball. J Strength Cond Res 29: 3006-3015, 2015.

31. Schelling X, Torres L. Accelerometer load profiles for basketball-specific drills in elite players. J Sports Sci Med 15: 585-591, 2016.

32. Spiteri T, Nimphius S, Hart NH, et al. Contribution of strength characteristics to change of direction and agility performance in female basketball athletes. J Strength Cond Res 28: 2415-2423, 2014.

33. SPSS Data Analysis: Kolmogorov-Smirnov Tests. Available at: https:// www.spss-tutorials.com/spss-kolmogorov-smirnov-test-for-normality/. Accessed June 10, 2019.

34. Stojanovic E, Stojiljkovic N, Scanlan AT, et al. The activity demands and physiological responses encountered during basketball match-play: A systematic review. Sports Med 48: 111-135, 2018.

35. Svilar L, Castellano J, Jukic I. Load monitoring system in top-level basketball team: Relationship between external and internal training load. Kinesiology 50: 25-33, 2018.

36. Svilar L, Castellano J, Jukic I, Casamichana D. Positional differences in elite basketball: Selecting appropriate training- load measures. Int J Sports Physiol Perform 13: 947-952, 2018.

37. Swann C. Flow in sport. In: Flow Experience: Empirical Research and Applications. L. Harmat, FO Andersen, F Ullen, et al, eds.Switzerland: Springer International Publishing, 2016. pp. 51-64.

38. Wundersitz DW, Gastin PB, Robertson S, Davey PC, Netto KJ. Validation of a trunk-mounted accelerometer to measure peak impacts during team sport movements. Int J Sports Med 36: 742-746, 2015. 